\title{
Identifying and managing younger women at high risk of cardiovascular disease
}

\author{
Kajenny Srivaratharajah MD MSc, Beth L. Abramson MD MSc
}

Cite as: CMAJ 2019 February 11;191:E159-63. doi: 10.1503/cmaj.180053

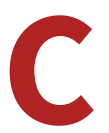

ardiovascular disease (CVD), which is largely preventable, is a leading cause of morbidity and mortality among Canadian women. For this article, we use CVD to refer to ischemic heart disease and stroke. Data for peripheral arterial disease in women are limited and are addressed elsewhere. ${ }^{1}$ Until now, risk reduction has focused largely on postmenopausal woman with traditional risk factors: diabetes, smoking, hypertension and hyperlipidemia. Consequently, CVD mortality has declined, largely driven by those aged 50 years and older. ${ }^{2} \mathrm{~A}$ recent study of 20-year temporal trends in admissions to hospital and deaths caused by atherosclerotic cardiovascular disease in Ontario reported that mortality rates for circulatory diseases in women declined $52.8 \%$ between 1994 and 2012. ${ }^{3}$ However, annual rates of decline were least evident in individuals younger than 50 years of age, suggesting that CVD among younger adults remains a cause for concern. ${ }^{3}$ The lowest rate of decline in CVD-related mortality and, in some cases, an increase in CVD-related admissions to hospital and mortality have been observed in younger women. 3.4

Contemporary Canadian data suggest the gap in cardiovascular mortality between men and women may be closing. ${ }^{5}$ Yet young women with ST-segment elevation myocardial infarction (MI) have $15 \%-20 \%$ higher rates of death than men of similar age. ${ }^{6}$ Whether this is related to systematic differences in care or true biological differences, or a combination, is unclear. What is clear is that addressing cardiovascular health in women younger than 50 years of age requires thinking beyond traditional risk factors in primordial prevention.

We present a brief overview of sex differences in traditional cardiovascular risk factors and a focused review of key nontraditional risk factors in younger women (i.e., ovarian dysfunction, infertility, reproductive therapies and pregnancy complications). Our approach to gathering evidence is outlined in Box 1 .

\section{What is the effect of traditional cardiovascular risk factors in young women?}

A 2018 study concluded that the strongest predictor of acute coronary syndrome in women under the age of 45 years is diabetes (odds ratio [OR] 6.66, 95\% confidence interval [CI] 3.47-12.74),

\section{KEY POINTS}

- Pregnancy-related vascular complications, such as preeclampsia, need to be factored into risk assessment in younger women.

- Conditions such as premature ovarian dysfunction, use of reproductive therapies and infertility may increase long-term risk of cardiovascular disease.

- Early risk stratification and aggressive management of lifestyle may help mitigate future risk in premenopausal women with high-risk profiles.

- Long-term management strategies need to be defined in this population.

followed by hypertension (OR 4.30, 95\% Cl 3.42-5.38), hypercholesterolemia (OR 3.45 95\% Cl 2.60-4.29) and smoking (OR 1.63, $95 \% \mathrm{Cl} 1.34-1.98) .{ }^{7}$ This study also found that smoking was more prevalent and other traditional risk factors were less prevalent among young women compared with older women with acute coronary syndrome. ${ }^{7}$ The INTERHEART study identified diabetes, metabolic syndrome and tobacco use as stronger predictors of ischemic heart disease in women under the age of 50 compared with older women. ${ }^{8}$

Findings from a 1996 cohort study suggested that, when compared with men, smoking is a relatively stronger risk factor for $\mathrm{MI}$ in women less than 45 years of age (relative risk [RR] 7.1 in

\section{Box 1: Evidence used in this review}

We conducted a search of PubMed for articles published in English between July 2008 and July 2018. We used the Medical Subject Heading (MESH) terms "cardiovascular disease" and "young women" for our search. We narrowed the search further by searching for articles involving humans and adults aged 19 to 64 years. This resulted in 7671 hits, which were narrowed down to 6442 by excluding articles with the term "congenital heart defect." We reviewed the best matched first 300 articles. Relevant articles listed in the reference section of select articles were also reviewed. We discussed the highest level of evidence via randomized controlled trials when available, and where literature was limited, we used observational and case reports. 
women v. 2.3 in men). ${ }^{9}$ Diabetes is also associated with a higher RR for acute coronary syndrome among women than among men (RR 3.50 in women v. 2.06 in men). ${ }^{10}$ In addition, a multicentre prospective cohort study reported that young women with acute MI had more comorbidities and worse pre-event health status than men. ${ }^{11}$ A more recent review of this VIRGO study population found that $\mathrm{MI}$ with nonobstructive coronary arteries was 5 times more likely to occur in women than men and less likely to be associated with traditional risk factors. ${ }^{12}$ Similar results were reported in the GENESIS-PRAXY multicentre prospective cohort study involving adults between 18 and 55 years of age, with more nontraditional risk factors among women. ${ }^{13}$ With respect to sex differences in traditional risk factors, this study showed higher prevalence of diabetes, hypertension and family history of coronary artery disease among women. ${ }^{13}$

Contemporary data for sex and gender differences in traditional risk factors for stroke or cerebrovascular disease in young adults are limited. Based on observational data of ischemic stroke in individuals less than 50 years of age, hypertension, dyslipidemia and current smoking status are more frequent in men compared with women. ${ }^{14}$ However, this study also reported higher stroke severity on the Canadian Neurological Scale and overall unfavourable outcomes at discharge in women compared with men. A 2014 systematic review and meta-analysis on stroke and diabetes showed a higher RR in women than men less than 60 years of age. ${ }^{15}$

\section{Do estrogen and hormone replacement therapy affect cardiovascular disease risk?}

The incidence of CVD is lower in premenopausal women compared with men of similar age. ${ }^{16}$ First coronary event(s) occur, on average, 10 years later in women than men. ${ }^{16}$ Given that women appear to be mostly protected until mid-life, estrogen has been implicated as a protective factor. However, the cardioprotective role of estrogen is complex and not well understood. ${ }^{17}$ Estrogen has multiple effects on the cardiovascular system, including promotion of vasodilation, antioxidative defence and recovery from vascular injury, thereby reducing the development of atherosclerosis, and preventing cardiomyocyte and endothelial dysfunction. ${ }^{17}$ Despite theoretical benefits, trials examining the addition of treatment with estrogen after menopause have not shown a protective benefit for CVD. ${ }^{18} \mathrm{~A}$ recent analysis of data of 18 -year follow-up of participants from randomized trials suggests that postmenopausal hormone replacement therapy may not be harmful in women, however. ${ }^{19}$

\section{What is the relation between ovarian dysfunction and risk of ischemic heart disease?}

Younger women with ovarian dysfunction appear to have an increased risk of CVD. ${ }^{20,21}$ Women with premature ovarian failure have as much as $80 \%$ higher mortality from ischemic heart disease than those who go through menopause at the expected average age range of 49 to 55 years according to prospective research..$^{22}$ Endothelial dysfunction, dysglycemia, abnormal lipid profile and metabolic syndrome may be potential drivers of elevated risk in this subset of younger women. ${ }^{20,21}$ latrogenic (i.e., surgical and chemical) menopause before the age of 50 years also confers a similar increase in risk of CVD. ${ }^{23}$ In a small cohort study, hormone replacement therapy in these women was shown to improve endothelial function within 6 months of treatment; ${ }^{24}$ however, in those with premature ovarian failure, no long-term data on CVD outcomes are available.

Polycystic ovarian syndrome is a common endocrine disorder in premenopausal women, with prevalence ranging from $6 \%$ to $15 \% .{ }^{25} \mathrm{~A}$ review of studies involving women with polycystic ovarian syndrome found an increased risk of subclinical atherosclerotic disease, diabetes, dyslipidemia, obesity and endothelial dysfunction. ${ }^{25}$ Ten-year follow-up in postmenopausal women with polycystic ovarian syndrome or its characteristics did not show higher mortality or adverse cardiovascular events. ${ }^{26}$ However, there are methodological limitations to this study. Long-term, large-scale data for cardiovascular outcomes are lacking for this group, and this represents an area for future study.

\section{Does reproductive therapy increase women's risk of ischemic heart disease?}

Limited and conflicting research has examined long-term risk of CVD resulting from infertility and fertility treatments. A 2017 cross-sectional analysis involving women who completed the Framingham Heart Study Third Generation and Omni Cohort 2 Exam 2 (2008-2011) and reported infertility showed that selfreported infertility was associated with CVD risk factors such as elevated body mass index and waist circumference. ${ }^{27}$ However, an analysis of data from the Women's Health Initiative Observational Study did not find a history of infertility to be associated with coronary heart disease. ${ }^{28}$ A 2016 observational study involving women receiving infertility therapy found that failure of therapy was associated with $19 \%$ higher annual rates of cardiovascular events. ${ }^{29}$ Increased thromboembolic events in this group with failure of infertility therapy may explain the elevated future risk. ${ }^{29}$ Another possibility is that failure of treatment unmasks those with underlying endothelial dysfunction, a known risk factor for future CVD. A 2017 systematic review and meta-analysis of a small number of heterogeneous observational studies examining the association between reproductive therapies and CVD risk reported no increased rates of cardiovascular events. ${ }^{30}$ Further study is needed to clarify whether it is the state of infertility itself, or reproductive treatment, that is associated with future CVD. As the average age of child-bearing increases along with a rise in the number of women seeking reproductive therapy for infertility, this is a pressing question that must be addressed.

\section{What do pregnancy complications indicate about future risk of cardiovascular disease?}

Pregnancy is considered a natural "stress test" for maternal cardiovascular health. Maternal cardiometabolic abnormalities such as gestational diabetes and hypertensive disorders of pregnancy 
are manifestations of a "positive test." These maternal cardiometabolic disorders have been identified as risk factors for longterm cardiovascular disease. ${ }^{31-39}$

\section{Hypertensive disorders of pregnancy}

Hypertensive disorders of pregnancy occur in $2 \%-10 \%$ of pregnancies $^{31}$ and range in severity from gestational hypertension (hypertension after 20 weeks of gestation) to preeclampsia (hypertension after 20 weeks with end-organ damage with or without proteinuria), eclampsia (preeclampsia and seizure) and HELLP syndrome (hemolysis, elevated liver enzyme and low platelet count levels). Women who had maternal placental syndromes (defined as preeclampsia, gestational hypertension, placental abruption and placental infarction) in pregnancy showed a twofold increase in CVD compared with those with pregnancies without maternal placental syndrome, with a mean age of onset of 38 years in a population-based retrospective cohort study involving over 1 million women. ${ }^{32}$ Maternal placental syndromes occurred in $7 \%$ of deliveries and showed an incremental rise in risk of future CVD with an adjusted hazard ratio of 3.1 in maternal placental syndromes with poor fetal growth and 4.4 in maternal placental syndromes with intrauterine death, compared with 1.8 and 2.1 for gestational hypertension and preeclampsia, respectively. ${ }^{32}$ Findings from the GENESIS-PRAXY study showed a threefold increase in premature CVD in women with a history of preeclampsia. ${ }^{33}$

Maternal placental syndromes also predict prognosis and survival following cardiovascular disease. ${ }^{34}$ Maternal placental syndromes double the risk of death in women undergoing coronary revascularization and recurrence quadruples this risk. Mechanisms are complex; however, endothelial dysfunction is implicated in preeclampsia and may be the driver for long-term cardiovascular risk $^{31}$ (Figure 1).

\section{Maternal dysglycemia}

Gestational diabetes occurs in $3 \%-4 \%$ of pregnancies ${ }^{31}$ and is associated with maternal postpartum diabetes, metabolic syndrome and CVD. ${ }^{35}$ Women with gestational diabetes had a 15 -fold higher rate of subsequent type 2 diabetes over 8.5 years followup, with a median age at onset of 37 years. ${ }^{36}$ Long-term 30-year follow-up of these women showed higher prevalence of cardiovascular disease (15.5\% v. $12.4 \%$; adjusted OR 1.85 , 95\% Cl $1.21-$ $2.82)$, with presentation at a younger age $(45.5 \pm 2.2$ v. $52.5 \pm$ 11.9 years) independent of development of postpartum diabetes or metabolic syndrome. ${ }^{37}$ Another study involving women aged 29 to 49 years with live births between April 1994 and March 1997 in Ontario found a HR of 1.71 (95\% Cl 1.08-2.69) for CVD events in those with gestational diabetes over a median follow-up time of 11.5 years; however, this effect was less clear when adjusted for development of type 2 diabetes. ${ }^{38}$ In 2018, a Canadian retrospective cohort study involving over 1 million women showed an association between gestational diabetes and elevated ischemic heart disease (HR 1.23, 95\% Cl 1.12-1.36) and MI (HR 2.14, 95\% Cl $1.15-2.47$ ) as much as 25 years after the index pregnancy. ${ }^{39}$

\section{Other complications of pregnancy and risk of cardiovascular disease}

There is increased cardiovascular risk, both in the short and long term, following maternal placental syndromes. ${ }^{40}$ In addition to preeclampsia, these syndromes include placental infarction and

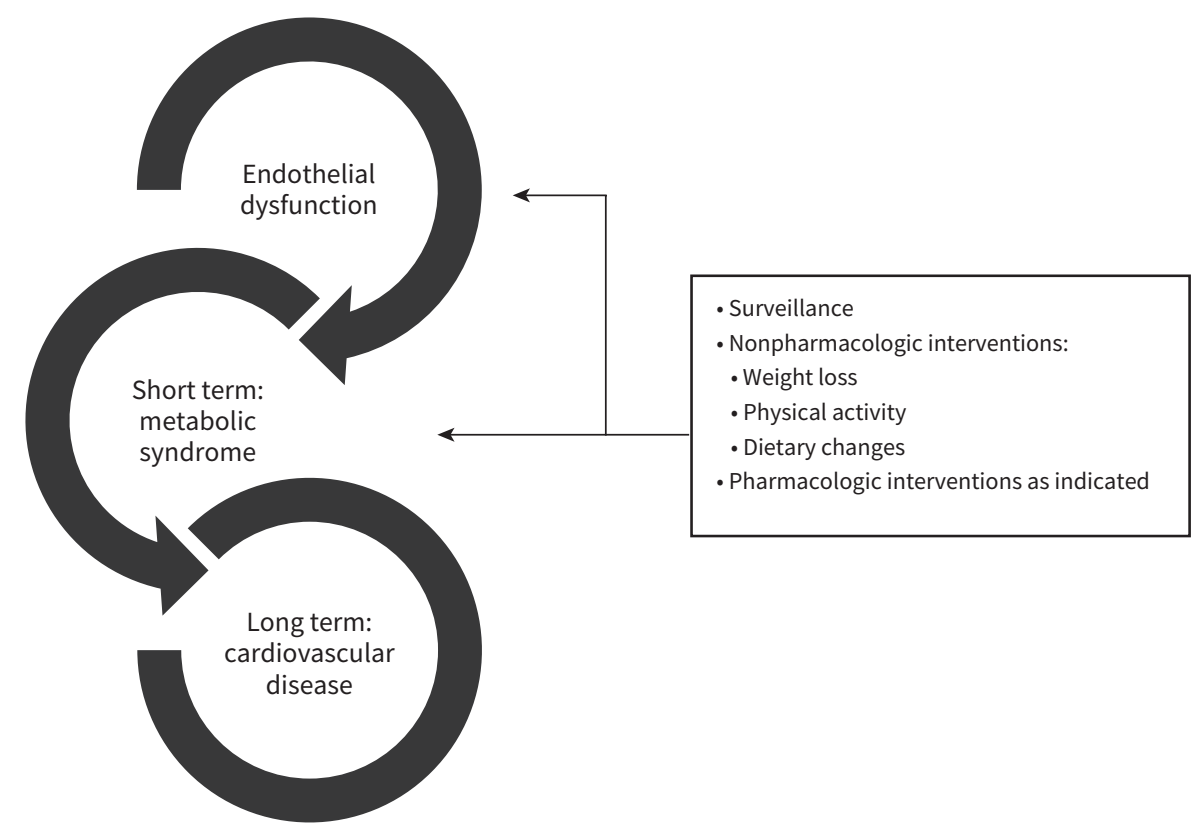

Figure 1: Cardiovascular disease cascade relating to nontraditional, sex-specific risk factors such as maternal placental syndromes (e.g., preeclampsia), premature ovarian failure, polycystic ovarian syndrome and infertility. 
abruption, both indicators of placental microvascular dysfunction. The outcome is often miscarriage or preterm delivery. In a large retrospective cohort study $(n=36713)$, women with any manifestation of this syndrome had a 19\% higher risk of CVD than pregnant women without maternal placental syndromes. Those with more than 1 manifestation of this syndrome had $40 \%$ higher risk of CVD within 4.9-year follow-up. Maternal placental syndromes in combination with preterm birth or small for gestational age increased risk of CVD by $45 \% .{ }^{40}$ Similarly, a 2017 population-based cohort study in Denmark found a 2.7-fold increase in CVD mortality and a 1.5-fold increase in morbidity among women with a history of placental abruption over long-term follow-up (median 18 years). ${ }^{41}$ Repeated miscarriages were also associated with future coronary heart disease (OR 1.99, 95\% $\mathrm{Cl} 1.13-3.50)$ but not cerebrovascular disease in a systematic review and meta-analysis of 10 studies involving over 500000 women with coronary heart disease and over 100000 with cerebrovascular disease. ${ }^{42}$

\section{How should premenopausal women at risk of cardiovascular disease be screened and managed?}

A challenge in risk assessment is addressing a subpopulation at elevated risk within a lower-risk group. Current risk assessment tools are largely based on age and traditional risk factors and tend to underestimate risk in certain groups of younger women who are at higher risk. The National Health and Nutrition Examination Survey reported that $82 \%$ of adults in the US (mean age 44 years) were categorized as having low short-term or 10-year risk, but two-thirds of this group were reclassified as having high lifetime risk. ${ }^{43}$ This disparity in short- and long-term classification was more prevalent in women. In younger adults, 30-year and lifetime CVD risk scores have shown predictive value. ${ }^{44}$ Whether the nontraditional risk factors we discussed in this article have independent additive predictive value in risk assessment of young women requires further study.

Young women and health care providers often lack understanding of nontraditional risk factors. This is complicated by the ambiguity in guidelines until recently. In 2011, the American Heart Association updated the guideline for the prevention of CVD in women and introduced pregnancy-related vascular complications in the CVD risk profile. ${ }^{45}$ Current evidence on CVD risk factors specific to women was reviewed in a 2016 American Heart Association Scientific Statement. ${ }^{16}$ The Canadian Cardiovascular Society guideline recently introduced the category of higher-risk younger women with hypertensive disorders of pregnancy in their screening recommendations. ${ }^{46}$

Based on these data and guidelines, we suggest that premenopausal women with both traditional and nontraditional risk factors for cardiovascular disease, as identified above, undergo early screening and close follow-up. Pharmacologic preventive therapy in addition to aggressive lifestyle management are indicated in those with risk factors. Pharmacologic therapy may include antihypertensive therapy, diabetes management, lipid management, medications for smoking cessation and estrogen replacement therapy in young women with premature ovarian dysfunction.

\section{Box 2: Unanswered questions}

- What are the ideal screening protocol and risk stratification strategies in higher-risk younger women?

- Which existing risk assessment scores are validated and calibrated to identify appropriately 10 -year and lifetime risk in these women?

- Which postpartum interventions are effective and, ultimately, have benefit in reducing long-term cardiovascular risk?

Possible suggestions for nonpharmacologic risk reduction include weight loss after gain in pregnancy, regular exercise and standard follow-up at 1 year postpartum to assess other cardiometabolic risks such as lipid profile and blood glucose levels. However, formal guidelines are lacking and further clarifying evidence is required to support such recommendations.

Management strategies are not all proven effective. An interdisciplinary, hospital-based postpartum clinic found no significant weight reduction or improvement in cardiometabolic risk despite improved physical activity, ${ }^{47}$ due, in part, to low levels of participation and dropout rates. Strategies to increase buy-in among young women, such as effective electronic health (e-health) interventions, need to be fostered. Use of e-health technologies to engage young mothers in weight-loss strategies has shown some benefit. ${ }^{48}$ Further study is warranted in this area.

\section{Conclusion}

Some younger women are at increased risk of CVD. Pregnancy complications and maternal placental syndromes present early markers of endothelial dysfunction. The postpartum period is an essential window of opportunity for risk stratification and early intervention to prevent long-term CVD. Premature ovarian dysfunction, reproductive therapies, and possibly infertility, can also be used to identify young women who may have elevated risk of future cardiovascular events. Long-term assessment and studies examining data on these women at higher risk are needed, and long-term management strategies need to be defined. Research questions to be addressed are summarized in Box 2. Increasing awareness of the interplay between both traditional and nontraditional cardiac risk factors in premenopausal women, greater focus in research on this topic and dissemination of practice guidelines with explicit screening and target measures, may help reduce the burden of CVD in younger women.

\section{References}

1. Srivaratharajah K, Abramson BL. Women and peripheral arterial disease: a review of sex differences in epidemiology, clinical manifestations, and outcomes. Can J Cardiol 2018;34:356-61.

2. Benjamin EJ, Virani SS, Callaway CW, et al.; American Heart Association Council on Epidemiology and Prevention Statistics Committee and Stroke Statistics Subcommittee. Heart disease and stroke statistics - 2018 update: a report from the American Heart Association. Circulation 2018;137:e67-492.

3. Tu JV, Khan AM, Ng K, et al. Recent temporal changes in atherosclerotic cardiovascular disease in Ontario: clinical and health systems impact. Can J Cardiol 2017;33:378-84.

4. Izadnegahdar M, Singer J, Lee MK, et al. Do younger women fare worse? Sex differences in acute myocardial infarction hospitalization and early mortality rates over ten years. J Womens Health (Larchmt) 2014;23:10-7. 
5. Pelletier R, Choi J, Winters N, et al.; GENESIS-PRAXY Investigators. Sex differences in clinical outcomes after premature acute coronary syndrome. Can J Cardiol 2016;32:1447-53.

6. Champney KP, Frederick PD, Bueno H, et al.; NRMI Investigators. The joint contribution of sex, age and type of myocardial infarction on hospital mortality following acute myocardial infarction. Heart 2009;95:895-9.

7. Bęćkowski M, Gierlotka M, Gąsior M, et al. Risk factors predisposing to acute coronary syndromes in young women $\leq 45$ years of age. Int J Cardiol 2018; 264:165-9.

8. Levit RD, Reynolds HR, Hochman JS. Cardiovascular disease in young women: a population at risk. Cardiol Rev 2011;19:60-5.

9. Njølstad I, Arnesen E, Lund-Larsen PG. Smoking, serum lipids, blood pressure, and sex differences in myocardial infarction. A 12-year follow-up of the Finnmark Study. Circulation 1996;93:450-6.

10. Huxley R, Barzi F, Woodward M. Excess risk of fatal coronary heart disease associated with diabetes in men and women: meta-analysis of 37 prospective cohort studies. BMJ 2006;332:73-8.

11. Dreyer RP, Smolderen KG, Strait KM, et al. Gender differences in pre-event health status of young patients with acute myocardial infarction: A VIRGO study analysis. Eur Heart J Acute Cardiovasc Care 2016;5:43-54.

12. Safdar B, Spatz ES, Dreyer RP, et al. Presentation, clinical profile, and prognosis of young patients with myocardial infarction with nonobstructive coronary arteries (MINOCA): results from the VIRGO study. J Am Heart Assoc 2018; 7:e009174.

13. Choi J, Daskalopoulou SS, Thanassoulis G, et al.; GENESIS-PRAXY Investigators. Sex-and gender-related risk factor burden in patients with premature acute coronary syndrome. Can J Cardiol 2014;30:109-17.

14. Martínez-Sánchez P, Fuentes B, Fernández-Domínguez J, et al. Young women have poorer outcomes than men after stroke. Cerebrovasc Dis 2011;31:455-63.

15. Peters SAE, Huxley RR, Woodward M. Diabetes as a risk factor for stroke in women compared with men: a systematic review and meta-analysis of 64 cohorts, including 775385 individuals and 12539 strokes. Lancet 2014;383:1973-80.

16. McSweeney JC, Rosenfeld AG, Abel WM, et al.; American Heart Association Council on Cardiovascular and Stroke Nursing, Council on Clinical Cardiology, Council on Epidemiology and Prevention, Council on Hypertension, Council on Lifestyle and Cardiometabolic Health, and Council on Quality of Care and Outcomes Research. Preventing and experiencing ischemic heart disease as a woman: state of the science: a scientific statement from the American Heart Association. Circulation 2016;133:1302-31.

17. Morselli E, Santos RS, Criollo A, et al. The effects of oestrogens and their receptors on cardiometabolic health. Nat Rev Endocrinol 2017;13:352-64.

18. Marjoribanks J, Farquhar C, Roberts H, et al. Long-term hormone therapy for perimenopausal and postmenopausal women. Cochrane Database Syst Rev 2017;1:CD004143.

19. Manson JE, Aragaki AK, Rossouw JE, et al.; WHI Investigators. Menopausal hormone therapy and long-term all-cause and cause-specific mortality: the Women's Health Initiative randomized trials. JAMA 2017;318:927-38.

20. Podfigurna-Stopa A, Czyzyk A, Grymowicz M, et al. Premature ovarian insufficiency: the context of long-term effects. J Endocrinol Invest 2016;39:983-90.

21. Yorgun H, Tokgözoğlu L, Canpolat U, et al. The cardiovascular effects of premature ovarian failure. Int J Cardiol 2013;168:506-10.

22. Jacobsen BK, Knutsen SF, Fraser GE. Age at natural menopause and total mortality and mortality from ischemic heart disease: the Adventist Health Study. J Clin Epidemiol 1999;52:303-7.

23. Rocca WA, Gazzuola-Rocca L, Smith CY, et al. Accelerated accumulation of multimorbidity after bilateral oophorectomy: a population-based cohort study. Mayo Clin Proc 2016;91:1577-89.

24. Kalantaridou SN, Naka KK, Papanikolaou E, et al. Impaired endothelial function in young women with premature ovarian failure: normalization with hormone therapy. J Clin Endocrinol Metab 2004;89:3907-13.

25. Gunning MN, Fauser BCJM. Are women with polycystic ovary syndrome at increased cardiovascular disease risk later in life? Climacteric 2017;20:222-7.
26. Merz CN, Shaw LJ, Azziz R, et al. Cardiovascular disease and 10-year mortality in postmenopausal women with clinical features of polycystic ovary syndrome. J Womens Health (Larchmt) 2016;25:875-81.

27. Mahalingaiah S, Sun F, Cheng JJ, et al. Cardiovascular risk factors among women with self-reported infertility. Fertil Res Pract 2017;3:7.

28. Parikh NI, Jeppson RP, Berger JS, et al. Reproductive risk factors and coronary heart disease in the Women's Health Initiative Observational Study. Circulation 2016;133:2149-58.

29. Udell JA, Lu H, Redelmeier DA. Failure of fertility therapy and subsequent adverse cardiovascular events. CMAJ 2017;189:E391-7.

30. Dayan N, Filion KB, Okano M, et al. Cardovascular risk following fertility therapy: systematic review and meta-analysis. J Am Coll Cardiol 2017;70:1203-13.

31. Nerenberg K, Daskalopoulou SS, Dasgupta K. Gestational diabetes and hypertensive disorders of pregnancy as vascular risk signals: an overview and grading of the evidence. Can J Cardiol 2014;30:765-73.

32. Ray JG, Vermeulen MJ, Schull MJ, et al. Cardiovascular health after maternal placental syndromes (CHAMPS): population-based retrospective cohort study. Lancet 2005;366:1797-803.

33. McDonald EG, Dayan N, Pelletier R, et al. Premature cardiovascular disease following a history of hypertensive disorder of pregnancy. Int J Cardiol 2016;219:9-13.

34. Ray JG, Booth GL, Alter DA, et al. Prognosis after maternal placental events and revascularization: PAMPER study. Am J Obstet Gynecol 2016;214:106.e1-14.

35. Burlina S, Dalfrà MG, Chilelli NC, et al. Gestational diabetes mellitus and future cardiovascular risk: an update. Int J Endocrinol 2016;2016:2070926.

36. Feig DS, Shah BR, Lipscombe LL, et al. Preeclampsia as a risk factor for diabetes: a population-based cohort study. PLoS Med 2013;10:e1001425.

37. Carr DB, Utzschneider KM, Hull RL, et al. Gestational diabetes mellitus increases the risk of cardiovascular disease in women with a family history of type 2 diabetes. Diabetes Care 2006;29:2078-83.

38. Shah BR, Retnakaran R, Booth GL. Increased risk of cardiovascular disease in young women following gestational diabetes mellitus. Diabetes Care 2008; 31:1668-9.

39. McKenzie-Sampson S, Paradis G, Healy-Profitós J, et al. Gestational diabetes and risk of cardiovascular disease up to 25 years after pregnancy: a retrospective cohort study. Acta Diabetol 2018;55:315-22.

40. Cain MA, Salemi JL, Tanner JP, et al. Pregnancy as a window to future health: maternal placental syndromes and short-term cardiovascular outcomes. Am J Obstet Gynecol 2016;215:484.e1-14.

41. Ananth CV, Hansen AV, Williams MA, et al. Cardiovascular disease in relation to placental abruption: a population-based cohort study from Denmark. Paediatr Perinat Epidemiol 2017;31:209-18.

42. Oliver-Williams CT, Heydon EE, Smith GC, et al. Miscarriage and future maternal cardiovascular disease: a systematic review and meta-analysis. Heart 2013;99:1636-44.

43. Marma AK, Berry JD, Ning H, et al. Distribution of 10 -year and lifetime predicted risks for cardiovascular disease in US adults: findings from the National Health and Nutrition Examination Survey 2003-2006. Circ Cardiovasc Qual Outcomes 2010;3:8-14.

44. Smith GN, Pudwell J, Walker M, et al. Ten-year, thirty-year, and lifetime cardiovascular disease risk estimates following a pregnancy complicated by preeclampsia. J Obstet Gynaecol Can 2012;34:830-5.

45. Mosca L, Benjamin EJ, Berra K, et al. Effectiveness-based guideline for the prevention of cardiovascular disease in women - 2011 update: a guideline from the American Heart Association. Circulation 2011;123:1243-62.

46. Anderson TJ, Grégoire J, Pearson GJ, et al. 2016 Canadian Cardiovascular Society guidelines for the management of dyslipidemia for the prevention of cardiovascular disease in the adult. Can J Cardiol 2016;32:1263-82.

47. Janmohamed R, Montgomery-Fajic E, Sia W, et al. Cardiovascular risk reduction and weight management at a hospital-based postpartum preeclampsia clinic. J Obstet Gynaecol Can 2015;37:330-7.

48. Sherifali D, Nerenberg KA, Wilson S, et al. The effectiveness of eHealth technologies on weight management in pregnant and postpartum women: systematic review and meta-analysis. J Med Internet Res 2017;19:e337.

\section{Competing interests: None declared.}

This article has been peer reviewed.

Affiliations: Division of General Internal Medicine (Srivaratharajah), Department of Medicine, McMaster University, Hamilton, Ont.; Division of Cardiology (Abramson), Department of Medicine, University of Toronto, Toronto, Ont.
Contributors: Both authors contributed equally to relevant literature review, manuscript writing and the revision process. Both authors gave final approval of the version to be published and agreed to be accountable for all aspects of this work.

Correspondence to: Beth Abramson, abramsonb@smh.ca 